\title{
The chemical evolution of the Galactic thick and thin disks
}

\author{
Cristina Chiappini ${ }^{1,2}$ \\ ${ }^{1}$ Geneva Observatory, Geneva University, 51 Chemin des Mailletes, Sauverny, CH1290, \\ Switzerland, email: Cristina.Chiappini@obs.unige.ch \\ ${ }^{2}$ Osservatorio Astronomico di Trieste - INAF, Via G. B. Tiepolo 11, Trieste, Italy
}

\begin{abstract}
Recent data have revealed a clear distinction between the abundance patterns of the Milky Way (MW) thick and thin disks, suggesting a different origin for each of these components. In this work we first review the main ideas on the formation of the thin disk. From chemical evolution arguments we show that the thin disk should have formed on a long timescale. We also show clear signs that the local stellar samples are contaminated by stars coming from inner radii. We then check what would have to be changed in such a model in order to explain the observables in the thick disk. We find that a model in which the thick disk forms on a much shorter timescale than thin disk and with a star formation efficiency of around a factor of 10 larger than that in the thin disk can account for the observed abundance ratio shifts of several elements between thick and thin disk stars. Moreover, the lack of scatter in the abundance ratio patterns of both the thick and thin disks suggest both components to have been formed in situ by gas accretion and not by mergers of smaller stellar systems. Especially for the thick disk, this last constraint becomes a strong one if its metallicity distribution extends to, at least, solar. Finally, we briefly discuss the interplay between present deuterium abundance and present infall rates in connection with the thin disk evolution.
\end{abstract}

Keywords. Galaxy: abundances, Galaxy: disk, Galaxy: evolution, stars: abundances

\section{Introduction}

Since Gilmore \& Reid (1983) it is known that our Galaxy has not only a thin disk, but also a thick disk component. Moreover, it has been found that thick disks are common in many other spiral galaxies (e.g. Yoachim \& Dalcanton 2008). For our Galaxy, there are striking differences between these two components, namely: a) thick disk stars are old (10-12 Gyrs) whereas thin disk stars are younger than 10 Gyrs; b) although they overlap in metallicity, their abundance patterns (i.e. $[\mathrm{X} / \mathrm{Fe}]$ vs. $[\mathrm{Fe} / \mathrm{H}]$ ) show important differences for $\mathrm{X}=\alpha$-elements, Mn, among other elements (see Feltzing, this volume), while being similar for most of the iron-peak elements; c) the thin disk is still forming stars whereas in the thick disk there is no more star formation or gas.

The question of how the thick and thin disk formed is intimately connected to the more general question of how galaxies form. As showed in this meeting one way to approach this problem is via galaxy formation simulations, starting with a cosmological model and computing the formation of galaxies in large scale. As beautifully summarized by S. White (this conference) this approach has led to an enormous progress, but still faces several problems such as uncertainties related to feedback and resolution. A stringent constraint upon models of galaxy formation is that of their chemical composition, which is directly linked to feedback. Here we use an alternative and complementary approach which has often been named the Archaeological Approach: we start from the present properties of the thick and thin disks in our Galaxy, for which detailed abundances are measured, and infer their past history. 


\section{The thin disk}

A robust result from chemical evolution models is that a closed-box model or a model in which metal-poor gas is accreted into the thin disk on very short timescales are not only incompatible with the G-dwarf metallicity distribution, but also with other observables such as the present star formation rate and the present deuterium abundance.
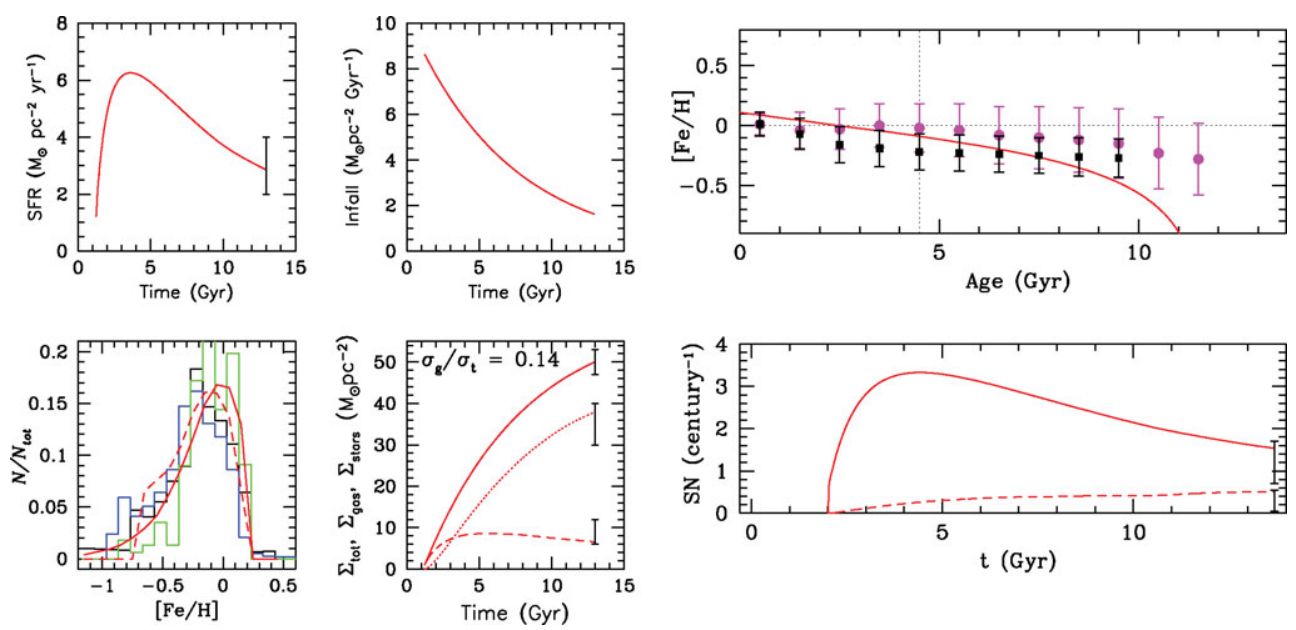

Figure 1. Best model predictions for the thin disk at the solar vicinity (see Chiappini et al. 1997). The vertical bars in some plots mark the observed value of the different quantities at present time at the solar vicinity. The data shown as histograms are from Rocha-Pinto \& Maciel (1996), Kotoneva et al. (2002) and Jørgensen (2000). For further details, see text.

Fig. 1 shows our best model predictions for the thin disk at the solar vicinity. The main input parameters are the infall rate $f(t)$ and the law for star formation, $\psi(t)$. We assume $f(t)=A e^{(-t / \tau)}$ (where $\tau=7 \mathrm{Gyrs}$ ) and $\psi(t)=B(R) \Sigma_{g}^{1.5}$, where $\mathrm{B}(\mathrm{R})$ is related to the star formation efficiency and the total mass density at each radii and $\Sigma_{g}$ is the gas surface mass density (see details in Chiappini et al. 1997). Note that the 7 Gyr timescale is compatible with recent cosmologically motivated infall (e.g. Naab \& Ostriker 2006, Colavitti et al. 2008 ). These assumptions ensure a good agreement with several observables.

Fig. 1 shows the most important ones, namely (from upper left to bottom right): a) the star formation history resulting from the adoption of the infall rate $(b), c)$ the age metallicity relation (AMR) compared with recent data by Soubiran et al. (2008 - squares) and da Silva et al. (2006 - dots); d) the G-dwarf metallicity distribution as measured by different groups (histograms) compared with the prediction of the second infall of Chiappini et al. (1997 - dashed line) and with the similar model present here, only for thin disk (solid line); e) the predicted variation of the total (solid), stellar (dotted) and gas (dashed) surface mass densities along $~ 11$ Gyrs of evolution and $\mathrm{f}$ ) the type II (solid) and Ia (dashed) supernovae rates. In addition, the model shown in Fig. 1 reproduces the solar and present-time abundances of the 28 elements included in our code.

Although we find good agreement for most of the observables, the model predicts a flatter AMR than observed, especially at larger ages. This is expected to happen for the following two reasons: a) the AMR for objects older than 10 Gyrs most probably includes thick-disk objects. In the case of Soubiran et al. thick-disk candidates were removed from their sample, which explains the better agreement of our model with this data-set; b) contamination of local samples with old metal-rich stars born in the inner regions of the 
disk (see Roskar, this conference). The latter effect seems to also happen. Fig. 2 (left panel) shows that although our model can well explain the $[\mathrm{Fe} / \mathrm{O}]$ trend with metallicity (which means that our enrichment timescales are correct), it stops at $[\mathrm{O} / \mathrm{H}] \sim 0.1$ dex. This is because, as said before, this model reproduces both the solar and the ISM oxygen abundances which are well constrained by observations.

Recent results have shown oxygen in young stars of the solar vicinity to be solar (see Chiappini et al. in prep and references therein). The similarity in the abundances of the Sun (4.5 Gyrs ago) and the ISM (now) can be understood in the framework of our model as due to the quite inefficient star formation rate in the last $4.5 \mathrm{Gyrs}$, leading to only a mild enrichment of the ISM since the formation of the Sun. However, the thin disk data shown in Fig. 2 extends up to at least $[\mathrm{O} / \mathrm{H}] \sim+0.3$ dex. We interpret this discrepancy as a clear indication that the local samples adopted to constrain chemical evolution models are contaminated by stars born at inner radii (see Grenon 1999).
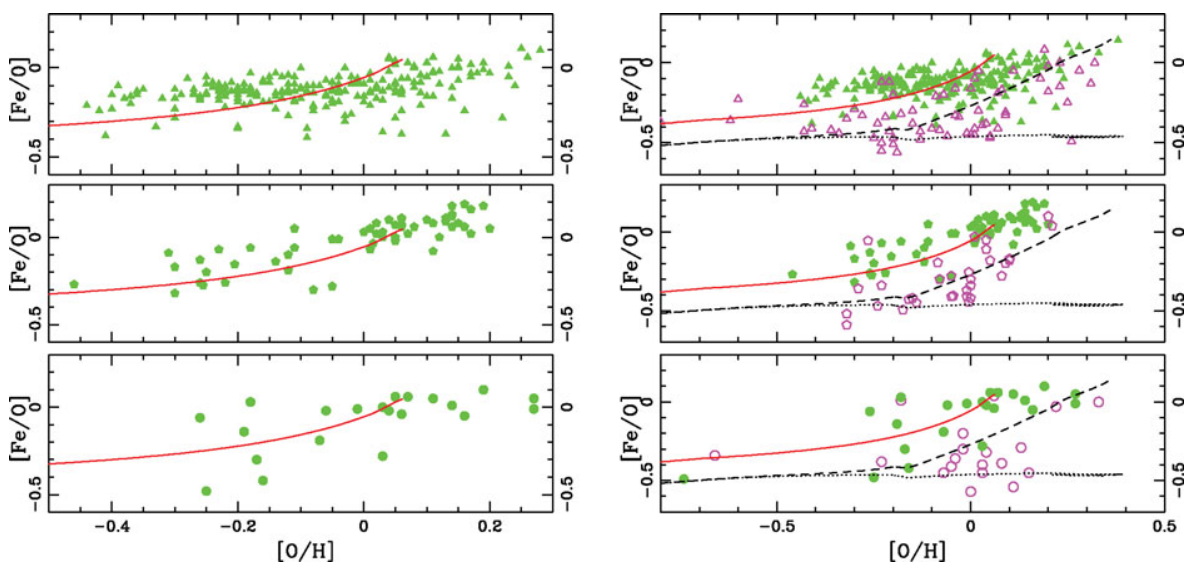

Figure 2. $[\mathrm{Fe} / \mathrm{O}]$ vs. $[\mathrm{O} / \mathrm{H}]$. Left panel: Filled symbols show thin disk stars observed by different groups using different oxygen lines and thus suffering from different uncertainties (Ramirez et al. (2007) - upper panel; Bensby et al. (2004) - middle panel and Mélendez et al. (2008) - lower panel). The solid line shows our best thin-disk model predictions (in both panels). Right panel: shows both thin disk (filled symbols) and thick disk stars (open symbols) measured by the same author to avoid systematic shifts. Our thick disk model with (dashed) and without (dotted) the contribution of SNIa is also shown.

\section{The thick disk}

For the thick disk, fewer constraints are available. Thick disk stars are older than $\sim 10$ Gyrs, and the metallicity distribution of this galactic component has a peak around $[\mathrm{Fe} / \mathrm{H}]=-0.5$, extending from $\sim-1.5$ to solar or above (the exact form of the metallicity distribution is still very uncertain, with large differences among different authors). The thick disk stars comprise 4 to $15 \%$ of the mass of the thin disk (Jurić et al. 2008).

The above properties support the idea that the star formation history of the thick disk was radically different from the one of the thin disk, being peaked towards earlier ages, hence implying a shorter timescale for the formation of this component in contrast to the extended formation of the thin disk. Here we present a thick-disk model similar to the one of the thin-disk but with two main differences: a much shorter infall timescale $\tau=0.4$ Gyr and a star formation efficiency larger than the one in the thin disk by a factor of 10. In this way we manage to get almost all stars in this component to have ages above 


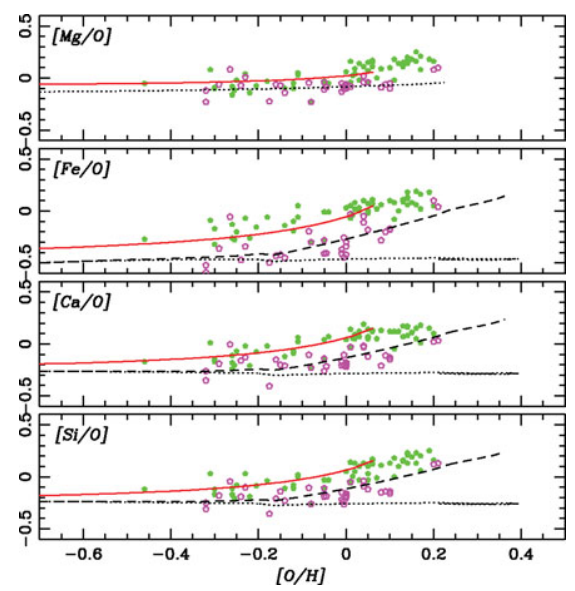

Figure 3. $[\alpha / \mathrm{O}]$ vs. $[\mathrm{O} / \mathrm{H}]$ for thin (filled symbols) and thick (open symbols) disk stars. The data are from Bensby \& collaborators. The theoretical predictions are shown for the thick disk (dashed line) and thin disk (solid line) models. Also shown is the case of a thick disk model with the SNIa (dotted line).

$\sim 10$ Gyr and at the same time a metallicity distribution peaking around $[\mathrm{Fe} / \mathrm{H}]=-0.5$ (Soubiran et al. 2008). In this model we assume, as for the thin disk, that the integral of the infall rate leads to the total mass density of the thick disk. However, not only is this number very uncertain, but the thick disk could also have started to form from much larger quantities of gas. In this case its star formation could have been halted not by a lack of gas (as in the model considered here) but due to the strong feedback produced by the intense star formation rate (see Elmegreen, this volume).

Fig. 2, right panel, shows the predictions of our thick-disk model for $[\mathrm{Fe} / \mathrm{O}]$ vs. $[\mathrm{O} / \mathrm{H}]$ (dashed line). The marked knee seen in this curve indicates the end of the star formation, when oxygen production stopped. At this point iron continues to increase due to type Ia supernovae (whose progenitors are low and intermediate-mass stars born before the halt in star formation). Also shown is a model without the SNIa contribution (dotted line) which obviously produces no up-turn. Notice that the reason for the up-turn seen in the thin disk curve is completely different. In this case, it is the larger contribution of SNIa with respect to SNII that increases the $[\mathrm{Fe} / \mathrm{O}]$ ratio with time (Time-delay model, see Matteucci 2001). Good agreement is also obtained for a large number of other abundance ratios such as $\mathrm{Mg} / \mathrm{O}, \mathrm{Ca} / \mathrm{O}, \mathrm{Si} / \mathrm{O}$ (see Fig. 3), Mn/O, among others (see Chiappini et al. in prep.). As clearly seen in Fig. 3, there is not only a simple shift between thick and thin-disk stars, but the differences among the two samples is a function of metallicity and it is well reproduced by our model. It can also be seen that the scatter in the abundance ratios is small.

A crucial point is that the abundance ratios of thick disk stars shown here exhibit trends with metallicity and are not just constant plateaus. However, it should be said that these trends become more evident if the thick disk really extends above solar metallicities. If this is the case, these trends can be interpreted as an indication of the coherent formation of the thick disk, challenging the idea that this component was formed from accreted stellar building blocks and favouring scenarios where the thick disk had an in situ formation, via gas accretion (similar to the one envisaged by Bournaud et al. 2007). Unfortunately, it is currently still debated if the stars with $[\mathrm{O} / \mathrm{H}]>0$ are really thick disk stars or are again stars coming from other galactic components such as thin-disk (other radii) or even bulge stars (Bensby et al. 2007, Ramirez et al. 2007). 
Finally, we notice a certain coincidence between the thick disk properties we infer here from our Archaeological Approach and the properties of the recently discovered disks at redshift $\sim 2$ (Pettini, this conference). These objects also seem to show high star formation efficiencies and to be actively forming their stars at times compatible with the star formation history we infer here for the thick disk.

\section{Infall and deuterium in the thin disk}

Up to now we have discussed models for the solar vicinity where most of the observational constraints are found. To satisfy other constraints such as the radial profiles of gas and star formation rate along the thin disk as well as the abundance gradients, we assume that the thin disk formed inside out (understood as shorter infall timescales in the inner regions relative to the outer ones - Chiappini et al. 2001). By integrating over several rings, we obtain the total present star formation and infall rates in the thin disk. Here we show the results for the model of Chiappini et al. (2001) (see Fig. 4). We see that this model predicts an infall rate of around $1 \mathrm{M}_{\odot} / \mathrm{yr}$ at $\sim 5 \mathrm{Gyrs}$ ago and a present rate of $\sim 0.4 \mathrm{M}_{\odot} / \mathrm{yr} \dagger$. Current estimates of the rate of infalling metal poor gas into the disk are $\sim 0.2 \mathrm{M}_{\odot} / \mathrm{yr}$ (Peek et al. 2007), but some extra contribution could still come from the Magellanic Clouds or other hidden sources (Fraternali, this conference).

For the integrated star formation rate in the disk we predict that it was around a factor of 5 larger $\sim 8$ Gyrs ago, than at present time. Interestingly, Bell (2008) found a similar result by studying the star formation rates obtained with Spitzer for spiral galaxies at redshifts $<1$ (lookback times corresponding to $\sim 8$ Gyrs ago).

As mentioned before, infall is a solution not only to the G-dwarf problem, but also helps to explain the present quantities of deuterium in the ISM. Deuterium is a very sensitive chemical marker of the gas consumption in a given locale. All of the deuterium in the Universe was created in the Big Bang. Stars destroy deuterium, so mass-loss from stars reduces the D abundance in the ISM, while accretion of pristine material from intergalactic space increases it. Thanks to infall, chemical evolution models for the solar vicinity that reproduce the major observational constraints predict only moderate $\mathrm{D}$ depletion, by at most a factor of 1.5 (e.g. Tosi et al. 1998, Chiappini et al. 2002). This was considered to be in good agreement with the observed values in the ISM.

With the new FUSE results, showing a large scatter in $[\mathrm{D} / \mathrm{H}]$ in the ISM, the interpretation of the data becomes complex (see Steigman et al. 2007 for a critical discussion). Linsky et al. (2006) prefer the largest values of D (the low ones attributed to depletion into dust grains) and conclude that $[\mathrm{D} / \mathrm{H}]=2.31 \pm 0.24 \times 10^{-5}$, which is smaller than the primordial value by only a factor $\sim 1.2$, in conflict with the predictions of chemical evolution models. According to Steigman et al. (2007) the real value can be slightly larger and still compatible with current models (Romano et al. 2006).

A possible way to alleviate the problem is to imagine larger quantities of infalling gas in the thin disk at present time. Right now this seems not to be possible because, as discussed above, the predicted infall rates are already on the limit of the observed values. On the other hand, Fraternali (this conference) presented some examples where the real infall rates are probably much larger than what is estimated from observed infalling gas clouds. If this was the case also for the MW, we would immediately alleviate the deuterium problem. It remains to be seen if larger quantities of infalling gas would still

$\dagger$ In that model the age of the thin disk was $\sim 12$ Gyrs. If we assume the disk is only 10 Gyrs old, then the present infall rate will be slightly larger, although the exact value depends on the adopted disk scale-length and total central mass density. 

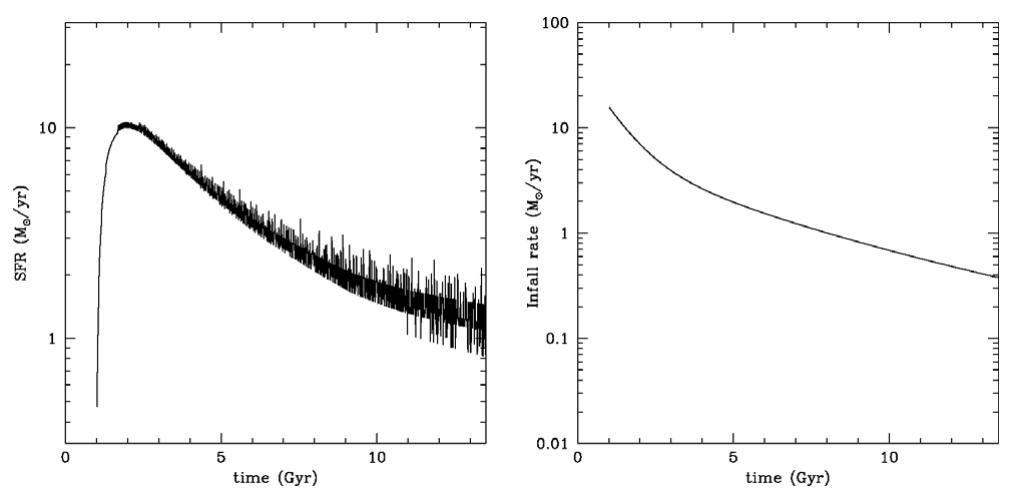

Figure 4. Predicted star formation and infall rates in the whole thin disk. This figure was obtained by integrating the several $2 \mathrm{kpc}$ rings of the model of Chiappini et al. (2001).

lead to models in good agreement with constraints such as the present gas surface density and present star formation rate.

\section{References}

Bell, E. 2008, Formation and Evolution of Galaxy Disks, eds. J. G. Funes \& E. M. Corsini Bensby, T., Zenn, A. R., Oey, M. S., \& Feltzing, S. 2007, ApJ, 663, 13

Bensby, T., Feltzing, S., \& Lundström, I. 2004, A\&A, 415, 155

Bournaud, F., Elmegreen, B. G., \& Elmegreen, D. M. 2007, ApJ, 670, 237

Chiappini, C., Renda, A., \& Matteucci, F. 2002, A\& $A$, 395, 789

Chiappini, C., Matteucci, F., \& Romano, D. 2001, ApJ, 554, 1044

Chiappini, C., Matteucci, F., \& Gratton, R. 1997, ApJ, 477, 765

Colavitti, E., Matteucci, F., \& Murante, G. 2008, A\& $A, 483,401$

da Silva, L., Girardi, L., Pasquini, L. et al. 2006, A\&A,458, 609

Gilmore, G. \& Reid, I. N. 1983, MNRAS, 202, 1025

Grenon, M. 1999, Astrophys. Space Science, 265, 331

Haywood, M. 2006, MNRAS, 371, 1760

Jørgensen, B. R. 2000, A\&A, 363, 947

Jurić, M., Ivezic, Z., Brooks, A. et al. 2008 astro-ph/0510520

Kotoneva, E., Flynn, C., Chiappini, C., \& Matteucci, F. 2002, MNRAS, 336, 879

Linsky, J. L., Draine, B. T., Moos, H. W. et al. 2006, ApJ, 647, 347

Matteucci, F., 2001 The chemical evolution of the Galaxy, Kluwer

Meléndez, J., Asplund, M., Alves-Brito, A. et al. 2008, A\& A, 484, L21

Naab, T. \& Ostriker, J. P. 2006, MNRAS, 366, 899

Peek, J. E. G., Putman, M. E., \& Sommer-Larsen, J. 2008, ApJ, 674, 227

Ramirez, I., Allende Prieto, C., \& Lambert, D. L. 2007, A\& $A, 465,271$

Romano, D., Tosi, M., Chiappini, C., \& Matteucci, M. 2006, MNRAS, 369, 295

Rocha-Pinto, H. J. \& Maciel, W. J. 1996, MNRAS, 279, 447

Soubiran, C., Bienaymé, O., Mishenina, T. V., \& Kovtyukh, V. V. 2008, A\&A A, 480, 91

Steigman, G., Romano, D., \& Tosi, M. 2007, MNRAS, 378, 576

Tosi, M., Steigman, G., Matteucci, F., \& Chiappini, C. 1998, ApJ, 498, 226

Yoachim, P. \& Dalcanton, J. J. 2008, ApJ, 682, 1004 